\title{
Ellipsoidal area mean gravity anomalies - precise computation of gravity anomaly reference fields for remove-compute-restore geoid determination
}

\section{Hirt}

Western Australian Centre for Geodesy \& The Institute for Geoscience Research Curtin University of Technology, GPO Box U1987, Perth, WA 6845, Australia Fax: +61 89266 2703; Email: c.hirt@curtin.edu.au

\section{S.J. Claessens}

Western Australian Centre for Geodesy \& The Institute for Geoscience Research Curtin University of Technology, GPO Box U1987, Perth, WA 6845, Australia Fax: +61 89266 2703; Email: s.claessens@curtin.edu.au

\begin{abstract}
Gravity anomaly reference fields, required e.g. in remove-compute-restore (RCR) geoid computation, are obtained from global geopotential models (GGM) through harmonic synthesis. Usually, the gravity anomalies are computed as point values or area mean values in spherical approximation, or point values in ellipsoidal approximation. The present study proposes a method for computation of area mean gravity anomalies in ellipsoidal approximation ('ellipsoidal area means') by applying a simple ellipsoidal correction to area means in spherical approximation. Ellipsoidal area means offer better consistency with GGM quasi/geoid heights. The method is numerically validated with ellipsoidal area mean gravity derived from very fine grids of gravity point values in ellipsoidal approximation. Signal strengths of (i) the ellipsoidal effect (i.e., difference ellipsoidal vs. spherical approximation), (ii) the area mean effect (i.e., difference area mean vs. point gravity) and (iii) the ellipsoidal area mean effect (i.e., differences between ellipsoidal area means and point gravity in spherical approximation) are investigated in test areas in New Zealand and the Himalaya mountains. The impact of both the area mean and the ellipsoidal effect on quasigeoid heights is in the order of several centimetres. The proposed new gravity data type not only allows more accurate RCR-based geoid computation, but may also be of some value for the GGM validation using terrestrial gravity anomalies that are available as area mean values.
\end{abstract}


Keywords: global geopotential model (GGM), remove-compute-restore (RCR) geoid computation, point gravity, area mean gravity, ellipsoidal area mean

\section{Introduction}

Regional gravimetric geoid and quasigeoid models are frequently computed using the remove-compute-restore (RCR) technique (e.g., Featherstone et al., 2004; Sjöberg, 2005). The basic idea of the RCR approach is the combination of terrestrial gravity observations and a global geopotential model (GGM), serving as a reference to provide the long- and mediumwavelength components of Earth's gravity field. In RCR-based computations, the GGMimplied gravity anomalies are subtracted from the terrestrial gravity observations, yielding a set of residual gravity anomalies ('remove'). These are transformed to residual quasi/geoid heights via Stokes's integral ('compute') and added to GGM-implied geoid heights ('restore'). Clearly, for precise application of the RCR technique, mutual consistency among the GGM-implied gravity anomaly field and quasi/geoid heights is an important prerequisite: The GGM-implied gravity anomaly field should be rigorously equivalent to the GGMimplied quasi/geoid heights. This is because any inconsistencies among these two fields contaminate the RCR quasi/geoid solution.

The present paper focuses on the accurate computation of the GGM gravity anomaly reference field. The computation of GGM quasigeoid heights is rather uncritical using the well-known Bruns equation (see e.g., Heiskanen and Moritz, 1967, p. 293). For subtleties in the computation of GGM geoid heights see, e.g., Rapp (1997) or Smith (1998). In terms of spatial representation and level of approximation, the computation of GGM gravity anomalies from spherical harmonic synthesis is ambiguous (see also details in Section 2):

First, GGM gravity anomalies can either be computed at discrete locations (point gravity) or as mean values over small area elements such as $1 \mathrm{~min} \times 1$ min cells (area mean gravity). The difference between area mean and point gravity anomalies is herein called the area mean effect. In Stokesian integration, the (continuous) gravity anomaly field is usually approximated by an equidistant grid composed of small area elements of stepwise-constant gravity anomalies. Often, area mean gravity anomalies are considered to be a better representation of average gravity across an area element than point gravity anomalies (see also Heiskanen and Moritz, 1967, p. 118). Also, because terrestrial gravity is commonly 
prepared in terms of area mean values (e.g., Featherstone et al., 2001, Claessens et al., 2011), the GGM gravity anomaly field should be provided in the same way. Naturally, GGM point gravity anomalies, subtracted from area mean terrestrial gravity, would introduce inconsistencies in the remove step of a RCR quasi/geoid computation.

Second, GGM gravity anomalies from spherical harmonic synthesis can be computed either in spherical approximation or ellipsoidal approximation. The term ellipsoidal effect is used here to denote the difference between gravity anomalies in ellipsoidal and spherical approximation. Ellipsoidal approximation is the more rigorous way for computation of GGM gravity anomalies, so as to avoid one spherical approximation effect in the quasi/geoid computation (e.g., Claessens, 2006). In other words, GGM ellipsoidal gravity anomalies approximate observed gravity anomalies more closely than those in spherical approximation.

The ellipsoidal effect of gravity anomalies has been studied by, e.g., Jekeli (1981), Cruz (1986), Gleason (1988), Vaníček et al. (1999) and Hipkin (2004), and is sometimes also called "the ellipsoidal correction to the spherical approximation". It should be noted that in the derivations of Jekeli (1981) and Cruz (1986), the ellipsoidal correction is split into two separate contributions that compensate for the fact that: 1) the partial derivative along the ellipsoidal normal is approximated by a partial derivative in the radial direction, and 2) that in spherical approximation the generally applied Somigliana-Pizetti reference gravity field (a spheroidal reference field) is approximated by an isotropic reference field (e.g., Heck, 1991). These two contributions are combined into one ellipsoidal correction here. Vaníček et al. (1999) use two ellipsoidal corrections too, but in their derivation the first of the two corrections also includes a so-called deflection error (Claessens 2006). Therefore, the ellipsoidal corrections of Vaníček et al. (1999) are not exactly compatible with our definition.

Taking into account both aspects of gravity representation (point values versus area means and spherical versus ellipsoidal approximation), it is desirable to compute the GGM gravity anomaly field in terms of area mean values in ellipsoidal approximation. However, with algorithms implemented in state-of-the-art spherical harmonic synthesis software such as harmonic_synth (Holmes and Pavlis, 2008), GGM gravity anomalies can be computed either as spherically approximated point values or area mean values, or, as point values in ellipsoidal approximation. As an immediate consequence, either the ellipsoidal effect or the 
area mean effect will cause inconsistencies in the remove-step of RCR-based geoid computations.

The present study investigates the computation of a new gravity field representation, the area mean gravity anomaly in ellipsoidal approximation (herein abbreviated to ellipsoidal area means). Section 2 provides the necessary mathematical background to compute GGM gravity anomaly fields in different approximations and spatial representations. A novel yet simple approach to compute ellipsoidal area means is introduced in Section 3. The approach combines point gravity anomalies in ellipsoidal and spherical approximation and area mean gravity anomalies in spherical approximation. Numerical verification results of the ellipsoidal area mean computation approach are found in Section 4. Also, an analysis of the signal patterns and amplitudes of the area mean and ellipsoidal effect is presented in Section 4, allowing for a better understanding of both effects. For this purpose, the state-of-the-art GGM EGM2008 (Pavlis et al., 2008) is used as data source. Then, we study the impact of the different GGM gravity anomaly types on quasigeoid heights, using Stokesian integration with a deterministically modified kernel (Featherstone et al., 1998). Finally, conclusions are drawn in Section 5.

As an alternative strategy to using ellipsoidal area means in GGM reference fields, it is also possible to apply an ellipsoidal correction to the terrestrial gravity observations (Vaníček et al., 1999). Importantly, the ellipsoidal effect need to be accounted for only once: either in the terrestrial observations [ie., Vanićek et al. (1999) approach] or in the GGM gravity anomaly reference field, as is proposed here. In both instances, ellipsoidal corrections are best computed from a GGM. In the Vaniček et al. (1999) approach, the ellipsoidal corrections can be applied to gravity observations before area means are computed. However, it is potentially more efficient to directly compute area means of ellipsoidal gravity anomalies or ellipsoidal corrections from the GGM, which is the approach taken here. Our approach represents a strategy to account for the ellipsoidal effect and area mean effect on gravity anomalies at the same time.

\section{Computation of GGM reference gravity fields}


We assume that computation points (e.g., arranged in an equidistant grid) are given in terms of (ellipsoidal) geodetic coordinates latitude $\varphi$, longitude $\lambda$ on the ellipsoid surface, i.e., ellipsoidal height $h=0$. To evaluate spherical harmonic synthesis expansions, spherical polar coordinates ( $r$ distance between the computation point and geocentre, $\theta$ geocentric colatitude and $\lambda$ geodetic longitude) are required. For the transformation between geodetic and spherical coordinates we refer to the standard geodetic literature, e.g., Torge (2001); Jekeli (2006).

A global geopotential model (GGM), such as EGM2008 (Pavlis et al., 2008), provides a set of fully-normalised spherical harmonic coefficients $\bar{C}_{n m}, \bar{S}_{n m}$ along with the two modelspecific scaling parameters GM (geocentric gravitational constant) and $a$ (semi-major axis). We use the standard spherical harmonic series expansion to compute the disturbing potential $T$ (e.g., Torge, 2001, p. 215; Holmes and Pavlis, 2008):

$T(r, \theta, \lambda)=\frac{G M}{r} \sum_{n=2}^{n \max }\left(\frac{a}{r}\right)^{n} \sum_{m=0}^{n}\left(\overline{\delta C}_{n m} \cos m \lambda+\bar{S}_{n m} \sin m \lambda\right) \bar{P}_{n m}(\cos \theta)$

with $n$ denoting the degree and $m$ the order of the harmonic coefficients and nmax is the maximum degree of evaluation (2190 in case of EGM2008). $\bar{P}_{n m}(\cos \theta)$ are the fullynormalised associated Legendre functions (e.g., Torge, 2001, p. 71) which depend on the geocentric co-latitude $\theta$ of the computation point. The term $\overline{\delta C}_{n m}=\bar{C}_{n m}-\bar{C}_{n m}^{G R S}$ indicates a subtraction of the low-degree even zonal harmonics $\bar{C}_{n m}^{G R S}$ of the GRS80 (Geodetic Reference System 1980) reference gravity field from the $\bar{C}_{n m}$ zonal harmonic coefficients of EGM2008 (a detailed explanation is given by, e.g., Smith, 1998).

The point gravity anomaly in spherical approximation is obtained from the well-known fundamental equation of physical geodesy which relates the disturbing potential $T$ to gravity anomalies (e.g., Torge, 2001, p. 259):

$$
\Delta g_{s p h}=-\frac{\partial T}{\partial r}-\frac{2}{r} T
$$


The spherical approximation is evident here as the partial derivative of the disturbing potential $T$ is formed with respect to the normal direction of the sphere (radius $r$ ).

The point gravity anomaly in ellipsoidal approximation can be computed using the generalised fundamental equation of physical geodesy (Grafarend et al., 1999; Claessens, 2006, p. 39) with the higher-order terms neglected:

$\Delta g_{\text {ell }}=-\frac{\partial T}{\partial h}+\frac{1}{\gamma} \frac{\partial \gamma}{\partial h} T$

with $\gamma$ reference gravity (at the ellipsoid) and $\partial \gamma / \partial h$ the vertical gradient of the reference gravity, cf. Torge (2001, p. 110). Importantly, the partial derivative of the disturbing potential $T$ is in the direction of the ellipsoidal normal $h$. The quantity $\partial T / \partial h$ is computed as a function of the radial derivative $\partial T / \partial r$ and the co-latitudinal derivative $\partial T$ / $\partial \theta$ (Claessens, 2006, p. 89):

$\frac{\partial T}{\partial h}=\cos \phi \frac{\partial T}{\partial r}-\sin \phi \frac{\partial T}{r_{e} \partial \theta}$

with $r_{e}$ the ellipsoidal radius (i.e., distance from the computation point to the geocentre) and $\phi$ the difference between geocentric and geodetic co-latitude, cf. Claessens (2006, p. 18-20; p. 89). The analytical expressions for the computation of the derivatives $\partial T / \partial r$ and $\partial T / \partial \theta$ are found, e.g., in Holmes (2002, p. 16) or Wolf (2007, p. 10).

For the computation of area mean gravity anomalies in spherical approximation, average values $\bar{T}$ of the disturbing potential are needed. These can be computed via integration over small area elements (e.g., 5 min x 5 min spatial extension), after Wenzel (1985, p. 34), and Holmes (2002, p. 133):

$$
\bar{T}\left(\lambda_{W}, \lambda_{E}, \varphi_{N}, \varphi_{S}\right)=\frac{1}{\left(\lambda_{E}-\lambda_{W}\right)\left(\sin \varphi_{N}-\sin \varphi_{S}\right)} \int_{\lambda_{1}}^{\lambda_{2}} \int_{\varphi_{1}}^{\varphi_{2}} T(\theta, \lambda, 0) \cos \varphi d \varphi d \lambda
$$


Here, $\lambda_{W}, \lambda_{E}$ are the meridians and $\varphi_{N}, \varphi_{S}$ the geodetic parallels bounding the area element in Western, Eastern, Northern and Southern direction, respectively. Upon insertion of Eq. (1) into Eq. (5), the solution of integral equation (5) involves the integration of fully-normalised associated Legendre functions $\bar{P}_{n m}(\cos \theta)$ (cf. Paul, 1978), and integration of Fourier coefficients $\cos m \lambda, \sin m \lambda$ (cf. Wenzel, 1985, p. 141). A complete solution for evaluation of Eq. (5) is derived in Holmes (2002, chapter 7). Area means of the radial derivative of the disturbing potential can be found using the same integration of fully-normalised associated Legendre functions and Fourier coefficients.

Area mean gravity anomalies in spherical approximation are obtained through modification of Eq. (2), after Wenzel (1985, p. 34):

$$
\overline{\Delta g}_{s p h}=-\frac{\partial \bar{T}}{\partial r}-\frac{2}{r} \bar{T}
$$

Note that the bar is used here and in the remainder of this study to distinguish point and area mean gravity field quantities. The state-of-the-art spherical harmonic synthesis software harmonic_synth (Holmes and Pavlis, 2008) allows computation of GGM point gravity anomalies either in spherical approximation (Eqs. 1,2), in ellipsoidal approximation (Eqs. 1,3,4), or, as area means in spherical approximation (Eqs. 5,6). However, the capability to compute area mean gravity in ellipsoidal approximation is not implemented. This is because the co-latitudinal derivative $\partial T / \partial \theta$ present in Eq. (4) would require the integration of the derivatives of associated Legendre functions with respect to the co-latitude, which cannot be performed by application of Paul's (1978) algorithm. The next section suggests simple methods that can be used to calculate area mean gravity anomalies in ellipsoidal approximation.

\section{Strategies to compute ellipsoidal area means}

There are (at least) two simple ways capable of providing an estimate of area mean gravity anomaly values in ellipsoidal approximation. The first approach (herein called the three-gridapproach) is based on the idea to correct spherically approximated area mean gravity 
anomalies by the ellipsoidal effect (i.e., the difference of gravity in ellipsoidal and spherical approximation).

The second approach, herein called the fine-grid-approach, is based on the computation of a very fine grid of ellipsoidal point gravity anomalies (e.g., at a resolution that is, say, 100 times better than the desired resolution) and to average the fine grid to the target grid resolution, giving estimates of ellipsoidal area means. However, as GGM reference fields used in modern RCR-based geoid modelling often consist of several million grid points with a spatial resolution of 1 min (e.g., Claessens et al., 2011; Featherstone et al., 2011), such fine grids would have to be made up of some 100 million points at which the spherical harmonic expansions would have to be evaluated. We consider the related computational efforts too prohibitive for routine RCR-based geoid computation. Nevertheless, the fine-grid-approach is of value here, as it is capable of providing independent estimates of ellipsoidal area means over smaller areas (say, some square degrees), serving as a 'check' on the first approach.

We propose the three-grid-approach for ellipsoidal area mean computation. The method is based on the assumption that the ellipsoidal effect of GGM gravity anomalies is fairly independent of the spatial representation of the gravity (area mean or point values), provided that the area size is sufficiently small, say, a few arc minutes. In other words, we presume that the difference between area means in spherical and ellipsoidal approximation is almost equal to the difference between point values in spherical and ellipsoidal approximation

$\overline{\Delta g}_{\text {ell }}-\overline{\Delta g}_{s p h} \approx \Delta g_{\text {ell }}-\Delta g_{\text {sph }}$

with

$\overline{\Delta g}_{\text {ell }}=$ area mean value in ellipsoidal approximation (unknown),

$\overline{\Delta g}_{\text {sph }}=$ area mean value in spherical approximation (Eq. 6),

$\Delta g_{\text {ell }}=$ point value in ellipsoidal approximation (Eq. 3) and

$\Delta g_{s p h}=$ point value in spherical approximation (Eq. 2). 
From Eq. (7), a solution for the computation of ellipsoidal area means $\overline{\Delta g}_{\text {ell }}$ follows immediately:

$\overline{\Delta g}_{\text {ell }} \approx \overline{\Delta g}_{\text {sph }}+\left(\Delta g_{\text {ell }}-\Delta g_{\text {sph }}\right)=: \overline{\Delta g}_{\text {ell }}^{3 g r i d s}$

Estimates of ellipsoidal area means are obtained from three gravity grids (therefore 'threegrid-approach'), that are computed with the existing publicly available spherical harmonic synthesis software harmonic_synth (Holmes and Pavlis, 2008). Spherically approximated area mean values of gravity anomalies $\overline{\Delta g}_{s p h}$ are computed in a first step. In a second step, two further grids are computed, one gravity anomaly grid of point values in spherical approximation $\Delta g_{\text {sph }}$ and one in ellipsoidal approximation $\Delta g_{\text {ell }}$. The differences between both point value grids is applied as correction to the area mean value grid, yielding estimates of gravity anomaly area means in ellipsoidal approximation $\overline{\Delta g}_{\text {ell }}$. Confirmation of the validity of Eqs. (7) and (8) is obtained from a numerical validation experiment described in Section 4.1.

\section{Numerical tests}

\subsection{Verification of the three-grid-approach}

In order to demonstrate that the proposed three-grid-approach is capable of providing sufficiently precise estimates of ellipsoidal area means $\overline{\Delta g}_{\text {ell }}$, a numerical test was performed for a $2^{\circ} \times 2^{\circ}$ alpine test area located on the South Island of New Zealand (Fig. 1A). In this area, both the ellipsoidal effect and the area mean effect reach maximum values in the order of $0.5 \mathrm{mGal}$ (shown later). The test not only compares ellipsoidal area means $\overline{\Delta g}_{\text {ell }}$ from the fine-grid-approach and three-grid-approach, but also illustrates features of the ellipsoidal and area mean effect. The visualisation of gravity anomaly area means in spherical approximation $\overline{\Delta g}_{\text {sph }}$ gives an impression of the roughness of the gravity anomaly field in the $2^{\circ} \times 2^{\circ}$ test area (Fig. 1B).

Table 1 gives an overview of the GGM gravity anomaly grid computations performed with the harmonic_synth software (Holmes and Pavlis, 2008). Any of the grid computations use the current state-of-the-art GGM EGM2008 (Pavlis et al., 2008) in the spectral range of 
2..2160 and the zero-tide system. One grid provides gravity anomaly point values $\Delta g_{\text {ell }}$ on a very fine grid of 3" resolution. Because of (i) the fact that EGM2008 offers a resolution of $~ 5$ min of gravity field structures and (ii) the high resolution ( 21 x $21=441$ values per $1^{\prime}$ cell) of the fine grid, averaging of the fine grid into $1 \mathrm{~min} \times 1 \mathrm{~min}$ cells is considered yielding reasonable estimates of ellipsoidal area means. These can be used for verification of the three-grid-approach. Importantly, the boundaries of the $1 \mathrm{~min} x 1 \mathrm{~min}$ cells exactly match those of the spherically approximated area means $\overline{\Delta g}_{s p h}$ from Eq. (6).

The main features of the area mean effect (differences $\overline{\Delta g}_{s p h}-\Delta g_{s p h}$ ), cf. Fig. 2A, are highfrequency patterns with wavelengths of $\sim 20 \mathrm{~km}$ and amplitudes of up to 0.5-0.7 mGal (Table 2). The maximum amplitudes occur in those areas where the EGM2008 gravity anomaly field is locally maximum and minimum, these are the summit regions and depressions in the EGM2008 gravity anomaly field (compare with Fig 1B). In such regions, the point values do not closely enough approximate the $1 \mathrm{~min}$ area mean values. As such they are either underor overestimates of the area mean gravity values. Naturally, at coarser grid resolutions than 1 min, the area mean effect on gravity will become larger.

Fig. 2B shows the ellipsoidal effect (differences $\Delta g_{\text {ell }}-\Delta g_{s p h}$ ) in terms of point gravity values. The difference plot exhibits patterns of up to $0.6 \mathrm{mGal}$ with longer wavelengths than the area mean effect. The descriptive statistics of both effects are found in Table 2. In a 'classical treatment' of the reference gravity field, at least one of both effects would propagate into the residual gravity anomaly field, causing inconsistencies in RCR-based quasi/geoid computation.

The ellipsoidal area mean effect (differences among gravity anomaly area means $\overline{\Delta g}_{\text {ell }}$ in ellipsoidal approximation, as obtained from the three-grid-approach, and point gravity values $\Delta g_{\text {sph }}$ in spherical approximation) is shown in Fig. 3A. The depiction shows how the area mean and ellipsoidal effect superimpose, resulting in somewhat larger amplitudes than either effect individually (see also Table 2).

Finally, residuals among ellipsoidal area means from the three-grid-approach and the finegrid-approach

$\delta \overline{\Delta g}_{\text {ell }}=\overline{\Delta g}_{\text {ell }}^{\text {3grids }}-\overline{\Delta g}_{\text {ell }}^{\text {finegrid }}$ 
are shown in Fig. 3B. The residuals, which reflect a combination of the errors in both approaches, are found to be very small (RMS $0.002 \mathrm{mGal}$, maximum values of $0.015 \mathrm{mGal}$, cf. Table 2). This provides strong evidence of the correctness of both approaches to compute ellipsoidal area means. In addition, the good mutual agreement confirms our above assumption that the ellipsoidal effect is similar for area means and point values (cf. Section $3)$.

Because the verification experiment is restricted to one selected test area, it cannot be concluded that the method provides good enough accuracy everywhere on Earth. However, due to the topography present in our test area (see Fig. 1A) it is reasonable to assume that the proposed method does reach a similar performance in many other regions with comparable or less topography.

\subsection{Assessment of maximum signal strengths}

The second part of the numerical tests deals with assessment of the maximum signal strengths (i.e., amplitudes and RMS) of the ellipsoidal and area mean effect for different regions. We have selected test areas in New Zealand and in the Himalaya mountains. The ruggedness of the New Zealand test area is representative for many other rugged areas (e.g., European Alps) while the Himalaya area allows a 'worst case' assessment of the gravity field effects. For both test areas (boundaries are given in Table 3 caption), EGM2008 was used in the spectral range 2..2160 to compute gravity anomaly grids in terms of point values in spherical and ellipsoidal approximation (Eqs. 2,3) and area means in spherical and ellipsoidal approximation (Eqs. 6,8). The grid resolutions are $1 \mathrm{~min}, 2 \mathrm{~min}$ and $4 \mathrm{~min}$, so as to evaluate the impact of the sampling.

Table 3 reports the descriptive statistics of the area mean effect $\left(\overline{\Delta g}_{s p h}-\Delta g_{s p h}\right)$, the

ellipsoidal effect $\left(\Delta g_{\text {ell }}-\Delta g_{\text {sph }}\right)$, and the ellipsoidal area mean effect $\left(\overline{\Delta g}_{\text {ell }}-\Delta g_{\text {sph }}\right)$. Within the same test area, the ellipsoidal effect is fairly independent of the grid resolution used. The amplitudes are about $0.6 \mathrm{mGal}$ for the New Zealand test area and reach maximum values of $\sim 1.6 \mathrm{mGal}$ in the high Himalaya mountains. The RMS is found to be generally small $(\sim 0.1$ mGal for New Zealand and $\sim 0.2 \mathrm{mGal}$ for the Himalayas). A similar analysis has been published by Hipkin (2004) based on evaluation of EGM96 (Lemoine et al., 1998) to degree 360. Globally, Hipkin (2004) found values of the ellipsoidal effect ranging between -0.84 to 
$1.15 \mathrm{mGal}$ and a standard deviation of $0.07 \mathrm{mGal}$. The differences between Hipkin's and our figures reflect mainly the fact (i) that the spectral range of degree 361 to 2160 is not included in Hipkin’s values and (ii) the mountainous test areas used in our study.

As expected, the grid resolution strongly influences the amplitudes of the area mean effect. For New Zealand, the maximum amplitudes steadily increase from $\sim 0.7-0.8 \mathrm{mGal}$ (at $1 \mathrm{~min}$ ), $\sim 3 \mathrm{mGal}$ (at $2 \mathrm{~min}$ ) to the level of $\sim 10 \mathrm{mGal}$ at $4 \mathrm{~min}$ grid resolution. For the Himalaya mountains, the effects are even more pronounced ( $\sim \mathrm{mGal}, \sim 15 \mathrm{mGal}$ and $\sim 60 \mathrm{mGal}$ for 1 min, 2 min and 4 min grid resolution, respectively). This shows that the mean gravity anomaly across the area elements deviates stronger from point values with decreasing grid resolution.

Again, in the 'classical' treatment of the GGM gravity anomaly reference field, at least one of both effects would reduce the level of consistency of the gravity and height anomaly reference fields. Particularly with grid resolutions of $2 \mathrm{~min}$ (and coarser), the area mean effect reaches magnitudes, which may not be negligible in practice. Ellipsoidal area mean gravity values - as introduced in Section 3 - can be used to account for both effects at the same time.

\subsection{Effect on quasigeoid undulations}

\subsubsection{Use of NZGeoid09 observed gravity}

The third numerical test focuses on the propagation of the ellipsoidal effect and area mean effect of gravity anomalies into quasigeoid heights. New Zealand is selected as test area and a data set consisting of area means of terrestrial gravity anomalies $\overline{\Delta g}_{N Z}$ is used for this test. This data set was also used as input data for the computation of the recent gravimetric NZGeoid09 model of New Zealand (Claessens et al., 2011). It was interpolated from observed land and sea gravity to a 1 min x 1 min grid [see Claessens et al. (2011) for details of the computation procedure]. The New Zealand terrestrial gravity data set $\overline{\Delta g}_{N Z}$ is here utilised for a series of RCR-quasigeoid computations with the GGM gravity anomaly reference field prepared in four different ways (Sections 2 and 3): (i) point values in spherical

approximation $\Delta g_{s p h}$, (ii) area mean values in spherical approximation $\overline{\Delta g}_{s p h}$, (iii) point 
values in ellipsoidal approximation $\Delta g_{\text {ell }}$ and (iv) area mean values in ellipsoidal approximation $\overline{\Delta g}_{\text {ell }}$. In any of the four variants, EGM2008 was used as GGM reference model within spectral degrees 2..2160 (as was the case with the computation of NZGeoid09).

For the transformation of residual gravity anomalies (i) $\overline{\Delta g}_{N Z}-\Delta g_{s p h}$, (ii) $\overline{\Delta g}_{N Z}-\overline{\Delta g}_{s p h}$, (iii) $\overline{\Delta g}_{N Z}-\Delta g_{\text {ell }}$ and (iv) $\overline{\Delta g}_{N Z}-\overline{\Delta g}_{\text {ell }}$ to residual quasigeoid undulations $\zeta$, we used the Curtin in-house software FFT1Dmod. This software performs Stokesian integration using a deterministically modified kernel (Featherstone et al., 1998) and the 1D fast Fourier transform integration technique (Haagmans et al., 1993). The integration parameters of the Featherstone et al. (1998) modified kernel were chosen equal to those used in the computation of NZGeoid09: degree of modification $L=40^{\circ}$ and integration cap size $\psi_{0}=2.5^{\circ}$. See Claessens et al. (2011) for the optimisation tests that were performed as a justification of the parameter selection. The residual quasigeoid undulations $\zeta$ computed from $\overline{\Delta g}_{N Z}-\Delta g_{s p h}$ were then compared against the residual quasigeoid undulations $\zeta$ obtained from the three residual gravity data sets $\overline{\Delta g}_{N Z}-\overline{\Delta g}_{s p h}, \overline{\Delta g}_{N Z}-\Delta g_{\text {ell }}$ and $\overline{\Delta g}_{N Z}-\overline{\Delta g}_{\text {ell }}$, respectively. Consequently, the differences exhibit the impact of the GGM reference field variants on the results of RCR-based quasigeoid computations.

Fig. $4 \mathrm{~A}$ and $\mathrm{B}$ show the differences between the residual gravity fields $\overline{\Delta g}_{N Z}-\Delta g_{s p h}$ and $\overline{\Delta g}_{N Z}-\overline{\Delta g}_{s p h} \quad\left(=\right.$ area mean effect) and the differences between $\overline{\Delta g}_{N Z}-\Delta g_{s p h}$ and $\overline{\Delta g}_{N Z}-\Delta g_{\text {ell }}$ (= ellipsoidal effect). The two effect grids clearly differ in terms of spectral content, as already seen in Fig. 2A and 2B. The area mean effect (Fig. 4A) exhibits shortwavelength patterns while the ellipsoidal effect (Fig. 4B) features long- and mediumwavelength structures. The maximum signal strengths, however, are approximately the same for both effects.

The area mean effect on residual quasigeoid heights

$$
\delta \zeta_{N Z}^{a m}=\zeta\left(\overline{\Delta g}_{N Z}-\Delta g_{s p h}\right)-\zeta\left(\overline{\Delta g}_{N Z}-\overline{\Delta g}_{s p h}\right)
$$


varies between -4 and $3 \mathrm{~mm}$ (Fig. 4C, and Table 5). The ellipsoidal effect on the quasigeoid

$$
\delta \zeta_{N Z}^{e l l}=\zeta\left(\overline{\Delta g}_{N Z}-\Delta g_{s p h}\right)-\zeta\left(\overline{\Delta g}_{N Z}-\Delta g_{\text {ell }}\right)
$$

shows amplitudes between -12 and $31 \mathrm{~mm}$ (Fig. 4D, and Table 4). The ellipsoidal effect has a much stronger impact on the quasigeoid heights because of its medium-wavelength patterns: In Stokesian integration, even small amplitudes of $0.1-0.2 \mathrm{mGal}$ accumulate quickly to quasigeoid signals at the cm-level, because larger areas of cells are subject to similar gravity effects. The amplitudes of the ellipsoidal effect are significant for cm-quasigeoid modelling. Though the area mean effect is of little relevance with the grid resolution used here $(1 \mathrm{~min})$ it will exhibit larger amplitudes with coarser grid resolutions.

\subsubsection{Direct transformation of GGM gravity differences to quasigeoid heights}

As an alternative to the above experiment, we used FFT1Dmod for a direct transformation of

(i) area mean effect $\overline{\Delta g}_{s p h}-\Delta g_{s p h}$ (Fig. 4A) and (ii) the ellipsoidal effect $\Delta g_{\text {ell }}-\Delta g_{s p h}$ (Fig 4B) to quasigeoid heights $\zeta\left(\overline{\Delta g}_{s p h}-\Delta g_{s p h}\right), \zeta\left(\Delta g_{\text {ell }}-\Delta g_{s p h}\right)$, respectively. Using the same Stokesian integration parameters as before, the resulting quasigeoid heights are almost the same as the ones depicted in Figs 4C and 4D [The RMS of the differences $\zeta\left(\overline{\Delta g}_{s p h}-\Delta g_{s p h}\right)$ and $\delta \zeta_{N Z}^{a m}$ is $0.3 \mathrm{~mm}$ and those of the differences $\zeta\left(\Delta g_{\text {ell }}-\Delta g_{s p h}\right)$ and $\delta \zeta_{N Z}^{\text {ell }}$ is $\left.0.5 \mathrm{~mm}\right]$. Given that Stokesian integration is a linear mathematical operation, this result is within the expectations. It corroborates the correctness of the previous results in Section 4.3.1.

As a final test, we applied the Stokesian integration to all of the gravity anomaly differences listed in Table 3, yielding the quasigeoid equivalent of (i) the area mean effect $\zeta($ $\left.\overline{\Delta g}_{s p h}-\Delta g_{s p h}\right)$, (ii) the ellipsoidal effect $\zeta\left(\Delta g_{\text {ell }}-\Delta g_{s p h}\right)$ and (iii) the ellipsoidal area mean effect $\zeta\left(\overline{\Delta g}_{\text {ell }}-\Delta g_{\text {sph }}\right)$ for the test areas New Zealand and Himalayas at grid resolutions of 1, 2 and 4 min. From Table 5 it is seen that both the area mean and ellipsoidal effect can reach signal strengths at the cm-level in mountainous areas when a grid resolution of 2 min (or coarser) is used. With 1 min grids, the ellipsoidal quasigeoid effect is still at the cm-level, 
while the impact of the area mean effect on the quasigeoid heights may be negligible in practice.

It should be noted that the study by Hipkin (2004) also investigated how the ellipsoidal effect translates into quasi/geoid heights. In contrast to our study, Hipkin applied the method of spherical harmonic analysis to convert a global grid of the ellipsoidal effect on gravity anomalies into a spherical harmonic representation. He then converted the spherical harmonic coefficients of the ellipsoidal gravity effect to geoid heights (cf. Hipkin 2004, p. 176) and found amplitudes of $\sim 0.6-0.7 \mathrm{~m}$ and a standard deviation of $\sim 0.2 \mathrm{~m}$ (cf. Hipkin 2004, p. 177).

However, Hipkin's results are not in contradiction to the cm-amplitudes of the ellipsoidal quasigeoid effect reported in Table 5. Contrary to Hipkin (2004), our analysis and results are based on Stokesian integration using a modified integration kernel (Featherstone et al., 1998) along with a cap size $\psi_{0}=2.5^{\circ}$. The limitation of the integration area acts as a high-pass filter (cf. Vanićek and Featherstone, 1998) that reduces the influence of any long-wavelength signals in the gravity anomalies. Importantly, this procedure also reduces the ellipsoidal effect which possesses significant power in the long-wavelengths (cf. Hipkin, 2004, p. 176). In other words, Stokesian integration with modified kernels suppresses large parts of the ellipsoidal effect, which can be considered a desirable side effect. If Stokesian integration with the unmodified (original) Stokes's kernel (e.g., Torge 2001, p.282) and a cap size $\psi_{0}=180^{\circ}$ was used to convert a global grid of ellipsoidal gravity effect to quasi/geoid heights, the ellipsoidal effect would fully propagate into the quasi/geoid solution, akin to Hipkin's results.

\section{Conclusions and recommendations}

This study investigated different ways to compute gravity anomaly reference fields from a GGM and introduced a new approach for the computation of gravity anomaly area means in ellipsoidal approximation. This approach is based on the idea to correct area mean gravity anomalies in spherical approximation by the ellipsoidal effect. The proposed method, called the three-grid-approach, works because the difference among point gravity data in ellipsoidal and spherical approximation is largely independent of the data type (area mean or point value). The results were verified by ellipsoidal area mean values obtained from a very fine 
grid of point values of gravity anomalies in ellipsoidal approximation, that was sampled down and compared with results from the three-grid-approach. The mutual agreement was below $2 \mu \mathrm{Gal}$ (RMS), giving a strong indication of the high precision of both computations. The proposed approach to compute GGM gravity as ellipsoidal area means is straightforward from a computational perspective, solely requiring the computation of three gravity anomaly grids. This can be done by means of the publicly available spherical harmonic synthesis software harmonic_synth without modifying the code.

As a second aspect of this study, amplitudes and patterns of the ellipsoidal effect and area mean effect were analysed for regions with different topography, helping to assess signal strengths of both effects. It was found that the ellipsoidal effect may exhibit amplitudes of $\sim 0.5 \mathrm{mGal}$ for rugged terrain with maximum values of about $1.5 \mathrm{mGal}$ in the high Himalaya mountains. These values are largely independent of the grid resolution. The maximum amplitudes of the area mean effect and ellipsoidal effect are similar for 1 min grids. With coarser grid resolution, however, the area mean effect may exhibit signals of up to several mGal, depending on the ruggedness of the gravity field. While high-frequency patterns are the dominant feature of the area mean effect, long- and medium-wavelength structures prevail in the ellipsoidal effect grids.

Given the variety of error sources a gravimetric geoid computation may be affected by (e.g., Featherstone et al., 2001), a reference field preparation in terms of ellipsoidal point values will mostly be sufficient when high-resolution 1 min grids are used. It is the high grid resolution that keeps the impact of the area mean effect small. It was shown that - with 2 min grid resolution or coarser - both the area mean and the ellipsoidal effect may translate into quasigeoid effects at the cm-level in rugged terrain. In a 'classical' reference field preparation (either in terms of gravity area means in spherical approximation or point gravity in ellipsoidal approximation), at least one of the effects would propagate into the RCR quasigeoid solution. The proposed gravity representation 'ellipsoidal area means' accounts for the ellipsoidal and area mean effect at the same time. This avoids contamination of the gravity anomaly reference field in a systematic manner, by error patterns visible in Fig. 4.

The ellipsoidal area means approach may also be of some value for the validation of GGMs (e.g., space-collected models from recent satellite gravity field missions) from observed surface gravity if available in terms of area mean values. GGM ellipsoidal area mean gravity 
anomalies are expected to approximate area means of observed surface gravity more closely than any of the other three representations (GGM ellipsoidal point values, GGM spherical area means, or GGM spherical point values), allowing better GGM validation.

\section{Acknowledgements}

We thank the Australian Research Council for financial support through grant DP0663020 and GNS Science, Te Pü Ao, for providing a data set of terrestrial gravity observations over New Zealand. The figures were produced using the Generic Mapping Tools GMT (Wessel and Smith, 1998). We would like to thank the reviewers for their comments on this manuscript. This is The Institute for Geoscience Research (TIGeR) publication number XX. 


\section{References}

Claessens S.J., 2006. Solutions to Ellipsoidal Boundary Value Problems for Gravity Field Modelling, PhD thesis, Curtin University of Technology, Department of Spatial Sciences, Perth, Australia.

Claessens S.J., Hirt C., Amos M.J., Featherstone W.E. and Kirby J.F., 2011. The NZGEOID09 model of New Zealand. Survey Review, 43(319), 2-15, doi: 10.1179/003962610X12747001420780.

Cruz J.Y., 1986. Ellipsoidal corrections to potential coefficients obtained from gravity anomaly data on the ellipsoid. Report 371, Department of Geodetic Science and Surveying, The Ohio State University, Columbus.

Featherstone W.E., Evans J.D. and Olliver J.G., 1998. A Meissl-modified Vaníček and Kleusberg kernel to reduce the truncation error in gravimetric geoid computations. $J$. Geodesy, 72(3), 154-160, doi: 10.1007/s001900050157.

Featherstone W.E., Kirby J.F., Kearsley A.H.W., Gilliland J.R., Johnston G.M., Steed J., Forsberg R. and Sideris M.G., 2001. The AUSGeoid98 geoid model of Australia: data treatment, computations and comparisons with GPS-levelling data. J. Geodesy, 75(5-6), 313-330.

Featherstone W.E., Holmes S.A., Kirby J.F. and Kuhn M., 2004. Comparison of removecompute-restore and University of New Brunswick techniques to geoid determination over Australia, and inclusion of Wiener-type filters in reference field contribution. $J$. Surv. Eng., 130(1), 40-47.

Featherstone W.E., Kirby J.F., Hirt C., Filmer M.S., Claessens S.J., Brown N.J., Hu G. and Johnston G.M., 2011. The AUSGeoid09 model of the Australian Height Datum. J. Geodesy (online first), doi: 10.1007/s00190-010-0422-2.

Gleason D.M., 1988. Comparing ellipsoidal corrections to the transformation between the geopotential's spherical and ellipsoidal spectrums. Manuscripta Geodetica 13(2), 114129.

Grafarend E.W., Ardalan A. and Sideris M.G., 1999. The spheroidal fixed-free twoboundary-value problem for geoid determination (the spheroidal Bruns' transform). $J$. Geodesy, 73(1), 513-533. 
Haagmans R., de Min E. and van Gelderen M., 1993. Fast evaluation of convolution integrals on the sphere using 1D FFT, and a comparison with existing methods for Stokes' integral. manuscripta geodetica, 18: 227-241.

Heck B., 1991. On the linearized boundary value problems of physical geodesy, Report No. 407, Dept of Geod Sci and Surv, Ohio State University, Columbus.

Heiskanen W.A. and Moritz H., 1967. Physical geodesy, Freeman and Co., San Francisco.

Hipkin R., 2004. Ellipsoidal geoid computation. J Geodesy, 78(3), 167-179.

Holmes S.A., 2002. High-degree Spherical Harmonic Synthesis: New Algorithms and Applications, PhD thesis, Curtin University of Technology, Department of Spatial Sciences, Perth, Australia.

Holmes S.A. and Pavlis N.K., 2008. Spherical harmonic synthesis software harmonic_synth. (Online at: http://earth-info.nga.mil/GandG/wgs84/gravitymod/egm2008/index.html).

Jarvis A., Reuter H.I., Nelson A. and Guevara E. (2008), Hole-filled SRTM for the globe Version 4, Available from the CGIAR-SXI SRTM 90m database. (Online at http://srtm.csi.cgiar.org).

Jekeli C., 1981. The downward continuation to the Earth's surface of truncated spherical and ellipsoidal harmonic series of the gravity and height anomalies. Report 323, Department of Geodetic Science and Surveying, The Ohio State University, Columbus.

Jekeli C., 2006. Geometric Reference Systems in Geodesy. Report, Division of Geodesy and Geospatial Science, School of Earth Sciences, Ohio State University.

Lemoine F.G., Kenyon S.C., Factor J.K., Trimmer R.G., Pavlis N.K., Chinn D.S., Cox C.M., Klosko S.M., Luthcke S.B., Torrence M.H., Wang Y.M., Williamson R.G., Pavlis E.C., Rapp R.H. and Olson T.R., 1998. The development of the joint NASA GSFC and the National Imagery and Mapping Agency (NIMA) geopotential model EGM96. NASA/TP-1998-206861. National Aeronautics and Space Administration, Greenbelt.

Paul M.K., 1978. Recurrence relations for integrals of associated Legendre functions. Bulletin Geodesique, 52, 177-190.

Pavlis N.K., Holmes S.A., Kenyon S.C. and Factor J.K., 2008. An Earth Gravitational Model to Degree 2160: EGM2008. Presented at the 2008 General Assembly of the European Geosciences Union, Vienna, April 13-18, 2008.

Rapp R.H., 1997. Use of potential coefficient models for geoid undulation determinations using a spherical harmonic representation of the height anomaly/geoid undulation difference. J. Geodesy, 71(5), 282-289. 
Sjöberg L., 2005. A discussion on the approximations made in the practical implementation of the remove-compute-restore technique in regional geoid modelling. J. Geodesy, 78(11-12), 645-653.

Smith D.A., 1998. There is no such thing as “The” EGM96 geoid: Subtle points on the use of a global geopotential model. IGeS Bulletin, 8:17-28.

Torge W., 2001. Geodesy. 3rd Edition, de Gruyter, Berlin New York.

Vaníček P., Huang J., Novák P., Pagiatakis S., Véronneau M., Martinec Z., and Featherstone W.E., 1999. Determination of the boundary values for the Stokes-Helmert problem. $J$ Geodesy, 73(4), 180-192.

Vaníček P. and Featherstone W.E., 1998. Performance of three types of Stokes's kernel in the combined solution for the geoid. J Geodesy, 72(12), 684-697.

Wenzel H.-G., 1985. Hochauflösende Kugelfunktionsmodelle für das Gravitationspotential der Erde. Wissenschaftliche Arbeiten der Fachrichtung Vermesssungswesen der Universität Hannover Nr. 137 [in German].

Wessel P. and Smith W.H.F., 1998. New, improved version of the Generic Mapping Tools released. EOS Trans. AGU, 79, 579.

Wolf K.-I., 2007. Kombination globaler Potentialmodelle mit terrestrischen Schweredaten für die Berechnung der zweiten Ableitungen des Gravitationspotentials in Satellitenbahnhöhe. Wissenschaftliche Arbeiten der Fachrichtung Geodäsie und Geoinformatik an der Universität Hannover Nr. 264 [in German]. 


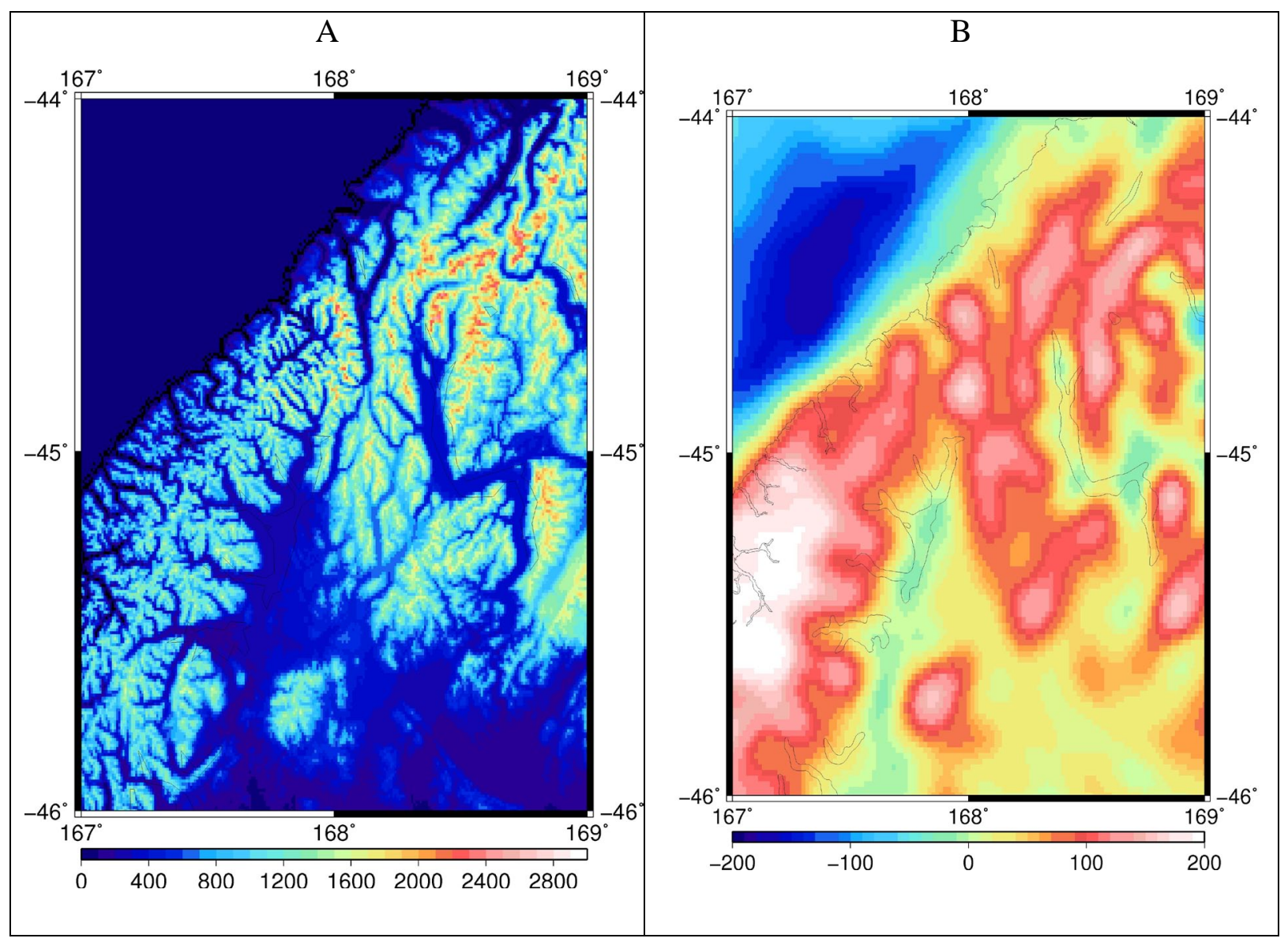

Fig. 1. A: Topography of the test area Southern Alps, New Zealand (from the Shuttle Radar Topography Mission SRTM global elevation model, cf. Jarvis et al., 2008) (units in m), B: EGM2008 area mean values $\overline{\Delta g}_{\text {sph }}\left(1^{\prime}\right.$ cells, degree 2..2160) in spherical approximation in the test area. (units in mGal). 


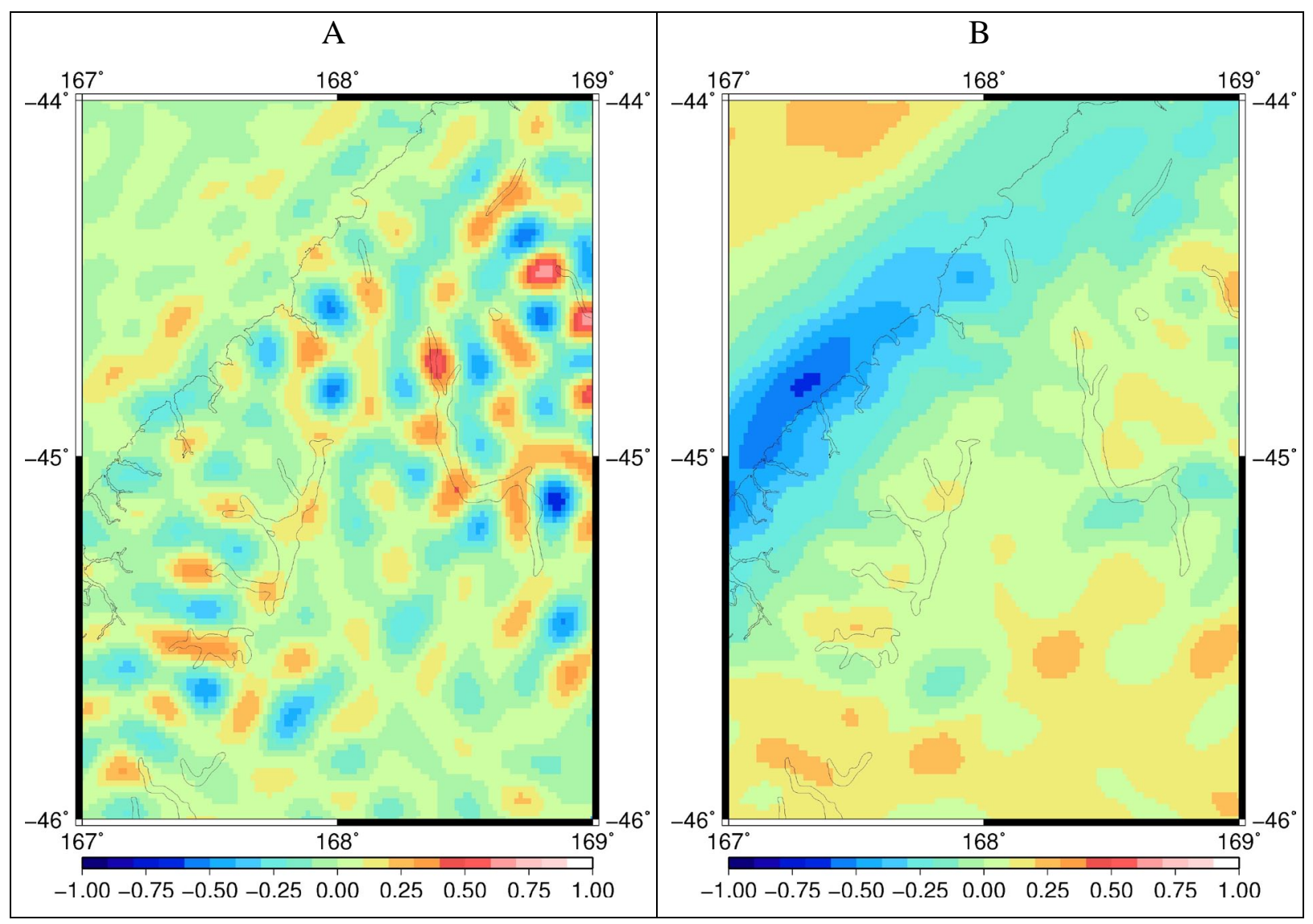

Fig. 2. A: Area mean effect (differences $\overline{\Delta g}_{s p h}-\Delta g_{s p h}$ between area mean values and point values of gravity anomalies, both in spherical approximation). B: Ellipsoidal effect (differences $\Delta g_{\text {ell }}-\Delta g_{s p h}$ among gravity anomalies in ellipsoidal and in spherical approximation, both in terms of point values). Units in mGal. 


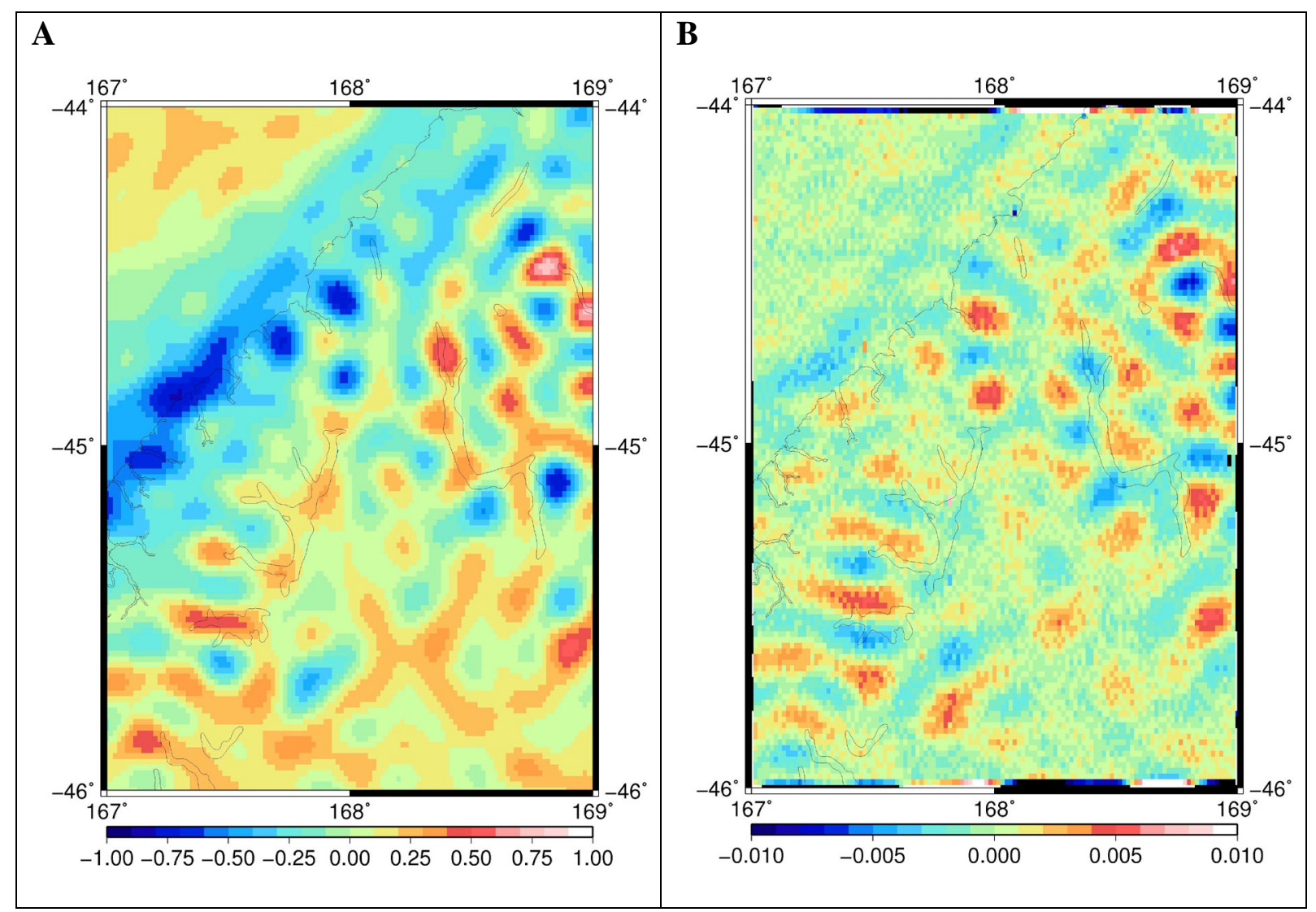

Fig. 3. A: Ellipsoidal area mean effect (differences $\overline{\Delta g}_{\text {ell }}-\Delta g_{\text {sph }}$ ). B: Differences $\overline{\Delta g}_{\text {ell }}$ (three-grid- approach) MINUS $\overline{\Delta g}_{\text {ell }}$ (fine-grid-approach). Units in mGal. Note the different scales used for panel A and B. 


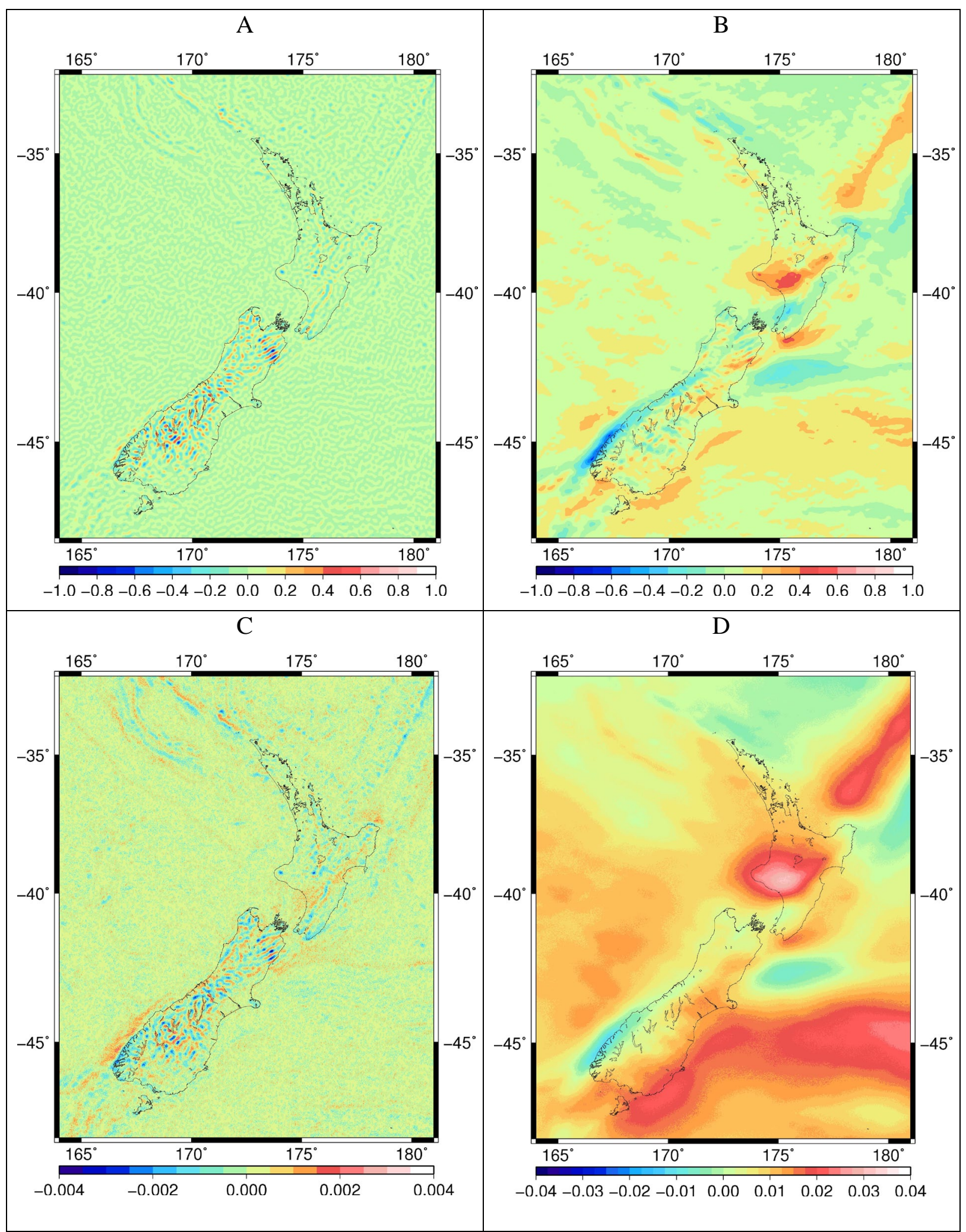

Fig. 4. A: area mean gravity effect [= residual gravity anomaly field differences $\overline{\Delta g}_{N Z}-\Delta g_{s p h}$ MINUS $\left(\overline{\Delta g}_{N Z}-\overline{\Delta g}_{s p h}\right)$ ]; B: ellipsoidal gravity effect [= residual gravity anomaly field differences $\overline{\Delta g}_{N Z}-\Delta g_{s p h}$ $\operatorname{MINUS}\left(\overline{\Delta g}_{N Z}-\Delta g_{\text {ell }}\right)$ ]; C: area mean quasigeoid effect $\delta \zeta_{N Z}^{a m}$ D: ellipsoidal quasigeoid effect $\delta \zeta_{N Z}^{\text {ell }}$. Units in $\mathrm{mGal}$ in panels $\mathrm{A}$ and $\mathrm{B}$, and $\mathrm{m}$ in panels $\mathrm{C}$ and $\mathrm{D}$. 
Table 1. Grid specifications for the verification of the three-grid-approach in the computation area bounded by $167^{\circ} \leq \lambda \leq 169^{\circ}$ and $-46^{\circ} \leq \varphi \leq-44^{\circ}$.

\begin{tabular}{|l|l|l|l|l|}
\hline Gravity type & Eq. & res & Number of points & Required for \\
\hline$\Delta g_{\text {ell }}$ & $(3)$ & $3^{\prime \prime}$ & $2401 \times 2401$ & fine-grid-approach \\
\hline$\Delta g_{\text {ell }}$ & $(3)$ & $1^{\prime}$ & $121 \times 121$ & three-grid-approach \\
\hline$\Delta g_{s p h}$ & $(2)$ & $1^{\prime}$ & $121 \times 121$ & three-grid-approach \\
\hline$\overline{\Delta g}_{s p h}$ & $(6)$ & $1^{\prime}$ & $121 \times 121$ & three-grid-approach \\
\hline
\end{tabular}

Table 2. Descriptive statistics of the area mean effect $\overline{\Delta g}_{s p h}-\Delta g_{s p h}$, the ellipsoidal effect $\Delta g_{e l l}-\Delta g_{s p h}$ (in terms of point values), the ellipsoidal area mean effect $\overline{\Delta g}_{\text {ell }}-\Delta g_{s p h}$ and the residual differences $\overline{\Delta g}_{\text {ell }}^{\text {grids }}$ (three-grid-approach) MINUS $\overline{\Delta g}_{\text {ell }}^{\text {finegrid }}$ (fine-grid-approach) in the computation area bounded by $167^{\circ} \leq \lambda \leq$ $169^{\circ}$ and $-46^{\circ} \leq \varphi \leq-44^{\circ}$ (units in mGal).

\begin{tabular}{|l|l|l|l|l|}
\hline Effect & Min & Max & Mean & RMS \\
\hline$\overline{\Delta g}_{s p h}-\Delta g_{s p h}$ & -0.671 & 0.681 & 0.0008 & 0.1446 \\
\hline$\Delta g_{\text {ell }}-\Delta g_{\text {sph }}$ & -0.617 & 0.298 & 0.0265 & 0.1806 \\
\hline$\overline{\Delta g}_{\text {ell }}-\Delta g_{\text {sph }}$ & -0.810 & 0.760 & 0.0274 & 0.2345 \\
\hline$\overline{\Delta g}_{\text {ell }}^{\text {3grids }}-\overline{\Delta g}_{\text {ell }}^{\text {finegrid }}$ & -0.018 & 0.014 & 0.0000 & 0.0016 \\
\hline
\end{tabular}


Table 3. Descriptive statistics of area mean effect $\overline{\Delta g}_{s p h}-\Delta g_{s p h}$, the ellipsoidal effect $\Delta g_{e l l}-\Delta g_{s p h}$ and and the ellipsoidal area mean effect $\overline{\Delta g}_{e l l}-\Delta g_{s p h}$ on GGM gravity anomalies in the New Zealand $\left(165^{\circ} \leq \lambda \leq\right.$ $185^{\circ}$ and $\left.-50^{\circ} \leq \varphi \leq-30^{\circ}\right)$ and Himalaya mountains $\left(80^{\circ} \leq \lambda \leq 100^{\circ}\right.$ and $\left.25^{\circ} \leq \varphi \leq 45^{\circ}\right)$ test areas at various grid resolutions (units in mGal).

\begin{tabular}{|c|c|c|c|c|c|c|c|}
\hline \multirow{2}{*}{$\begin{array}{c}\text { Grid } \\
\text { resolution }\end{array}$} & \multirow[t]{2}{*}{ Effect } & \multicolumn{3}{|c|}{ New Zealand } & \multicolumn{3}{|c|}{ Himalaya mountains } \\
\hline & & Min & Max & RMS & Min & Max & RMS \\
\hline \multirow[t]{3}{*}{$1 \mathrm{~min}$} & $\overline{\Delta g}_{s p h}-\Delta g_{s p h}$ & -0.88 & 0.71 & 0.04 & -4.18 & 3.72 & 0.32 \\
\hline & $\Delta g_{\text {ell }}-\Delta g_{\text {sph }}$ & -0.62 & 0.58 & 0.09 & -1.64 & 1.47 & 0.21 \\
\hline & $\overline{\Delta g_{e l l}}-\Delta g_{s p h}$ & -0.87 & 0.86 & 0.10 & -5.07 & 3.38 & 0.39 \\
\hline \multirow[t]{3}{*}{$2 \min$} & $\overline{\Delta g}_{s p h}-\Delta g_{s p h}$ & -3.48 & 2.78 & 0.14 & -16.49 & 14.53 & 1.27 \\
\hline & $\Delta g_{e l l}-\Delta g_{s p h}$ & -0.62 & 0.58 & 0.09 & -1.63 & 1.44 & 0.21 \\
\hline & $\overline{\Delta g}_{e l l}-\Delta g_{s p h}$ & -3.44 & 2.90 & 0.17 & -17.05 & 14.23 & 1.29 \\
\hline \multirow[t]{3}{*}{$4 \mathrm{~min}$} & $\overline{\Delta g}_{s p h}-\Delta g_{s p h}$ & -12.16 & 10.62 & 0.55 & -63.29 & 51.51 & 4.86 \\
\hline & $\Delta g_{e l l}-\Delta g_{s p h}$ & -0.62 & 0.55 & 0.09 & -1.62 & 1.40 & 0.21 \\
\hline & $\overline{\Delta g}_{e l l}-\Delta g_{s p h}$ & -12.17 & 10.74 & 0.56 & -63.85 & 51.09 & 4.87 \\
\hline
\end{tabular}

Table 4. Area mean effect, ellipsoidal effect and ellipsoidal area mean effect of the GGM-reference field on the quasigeoid heights. Statistics refers to land points of New Zealand; units in m.

\begin{tabular}{|l|l|l|l|l|}
\hline Effect & Min & Max & Mean & RMS \\
\hline Area mean effect $\delta \zeta_{N Z}^{\text {am }}$ & -0.004 & 0.003 & 0.000 & 0.001 \\
\hline Ellipsoidal effect $\delta \zeta_{N Z}^{\text {ell }}$ & -0.012 & 0.031 & 0.010 & 0.012 \\
\hline $\begin{array}{l}\text { Ellipsoidal area mean effect } \\
\zeta\left(\overline{\Delta g}_{N Z}-\Delta g_{s p h}\right)-\zeta\left(\overline{\Delta g}_{N Z}-\overline{\Delta g}_{\text {ell }}\right)\end{array}$ & -0.013 & 0.031 & 0.010 & 0.012 \\
\hline
\end{tabular}


Table 5. Descriptive statistics of area mean quasigeoid effect $\zeta\left(\overline{\Delta g}_{s p h}-\Delta g_{s p h}\right)$,, the ellipsoidal quasigeoid effect $\zeta\left(\Delta g_{\text {ell }}-\Delta g_{\text {sph }}\right)$ and the ellipsoidal area mean quasigeoid effect $\zeta\left(\overline{\Delta g}_{\text {ell }}-\Delta g_{s p h}\right)$ in the New Zealand and Himalaya mountains test areas at various grid resolutions (units in mm).

\begin{tabular}{|c|c|c|c|c|c|c|c|}
\hline \multirow{2}{*}{$\begin{array}{c}\text { Grid } \\
\text { resolution }\end{array}$} & \multirow[t]{2}{*}{ Effect } & \multicolumn{3}{|c|}{ New Zealand } & \multicolumn{3}{|c|}{ Himalaya mountains } \\
\hline & & Min & Max & RMS & Min & Max & RMS \\
\hline \multirow[t]{3}{*}{$1 \mathrm{~min}$} & $\zeta\left(\overline{\Delta g}_{s p h}-\Delta g_{s p h}\right)$ & -4 & 3 & 0.2 & -16 & 12 & 1.3 \\
\hline & $\zeta\left(\Delta g_{\text {ell }}-\Delta g_{s p h}\right)$ & -12 & 30 & 9.9 & -66 & 67 & 16.0 \\
\hline & $\zeta\left(\overline{\Delta g}_{e l l}-\Delta g_{s p h}\right)$ & -13 & 31 & 9.9 & -73 & 69 & 16.0 \\
\hline \multirow[t]{3}{*}{$2 \mathrm{~min}$} & $\zeta\left(\overline{\Delta g}_{s p h}-\Delta g_{s p h}\right)$ & -14 & 9 & 0.8 & -62 & 47 & 5.0 \\
\hline & $\zeta\left(\Delta g_{e l l}-\Delta g_{s p h}\right)$ & -12 & 30 & 9.9 & -66 & 67 & 16.0 \\
\hline & $\zeta\left(\overline{\Delta g}_{\text {ell }}-\Delta g_{\text {sph }}\right)$ & -18 & 33 & 9.9 & -112 & 84 & 16.8 \\
\hline \multirow[t]{3}{*}{$4 \mathrm{~min}$} & $\zeta\left(\overline{\Delta g}_{s p h}-\Delta g_{s p h}\right)$ & -51 & 35 & 3.1 & -239 & 172 & 19.4 \\
\hline & $\zeta\left(\Delta g_{e l l}-\Delta g_{s p h}\right)$ & -12 & 30 & 9.9 & -66 & 67 & 16.0 \\
\hline & $\zeta\left(\overline{\Delta g}_{e l l}-\Delta g_{s p h}\right)$ & -54 & 39 & 10.4 & -281 & 178 & 25.2 \\
\hline
\end{tabular}

